\title{
Percepción sobre la coordinación de la atención: el caso de las redes de servicios de salud de Xalapa y Veracruz, México, en el periodo 2014-2016*
}

\section{Perception of the Health Care Coordination: The Case of the Health Care Networks in Xalapa and Veracruz, Mexico during 2014-2016}

\section{Percepção sobre a coordenação da atenção: caso das redes de serviços de saúde de Xalapa e Veracruz, México, no período 2014-2016}

\begin{abstract}
Recepción: 27 de mayo de 2019. Aceptación: 28 de octubre de 2019.
\end{abstract}
Publicación: 30 de junio de 2020.

DOI: https://doi.org/10.11144/Javeriana.rgps19.pcac

\author{
Angélica Ivonne Cisneros Luján \\ Universidad Veracruzana, México \\ ORCID: https://orcid.org/0000-0002-7275-515X
}

Dulce María Cinta Loaiza

Universidad Veracruzana, México

ORCID: https://orcid.org/0000-0002-6389-6989

\author{
María Alejandra Sánchez Bandala ${ }^{a}$ \\ Universidad de la Sierra Sur, México \\ ORCID: https://orcid.org/0000-0002-4245-4743 \\ Vianey González Rojas \\ Secretaría de Salud, México \\ ORCID: https://orcid.org/0000-0002-3700-8546
}

\begin{abstract}
Cómo citar este artículo: Cisneros Luján AI, Cinta Loaiza DM, Sánchez Bandala MA, González Rojas V. Percepción sobre la coordinación de la atención: el caso de las redes de servicios de salud de Xalapa y Veracruz, México, en el periodo 2014-2016. Revista Gerencia y Políticas de Salud. 2020;19. https://doi.org/10.11144/ Javeriana.rgps19.pcac
\end{abstract}

\footnotetext{
a Autora de correspondencia. Correo electrónico: alejandra.bandala1@gmail.com
} 


\section{Resumen}

Una limitada coordinación asistencial impide garantizar una atención integrada, eficiente y de calidad. El objetivo del estudio fue explorar la percepción de profesionales de salud sobre la coordinación entre niveles de atención y los factores que la influyen. Se realizó un estudio cualitativo mediante el análisis de contenido de 54 entrevistas semiestructuradas y 11 grupos focales con personal de dos redes de servicios de salud en Xalapa y Veracruz, México. Los entrevistados refirieron dificultades para el seguimiento de los pacientes, desacuerdos respecto a los criterios de referencia, barreras de acceso a la atención especializada, baja calidad de los formatos de referencia, escasa recepción de los formatos de contrarreferencia y dificultades para seguir los circuitos de referencia. Atribuyeron estos problemas a la insuficiencia de recursos, la cobertura limitada del Seguro Popular, la sobrecarga de trabajo, falta de supervisión, deficiente formación de los médicos generales y limitada comunicación entre profesionales. Estos resultados coinciden con otros estudios en la región, por lo que se señala la necesidad de reforzar los mecanismos basados en la normalización, introducir estrategias basadas en la adaptación mutua y modificar rasgos de la estructura y cultura organizacional para lograr una coordinación de la atención efectiva, que contribuya a la integración de las redes.

Palabras clave: sistemas de salud, servicios de salud, calidad de la atención de salud, continuidad de la atención al paciente, investigación cualitativa.

\section{Abstract}

A limited coordination in the health care hinders the provision of a comprehensive, efficient, and good-quality health care. This study aims to explore how the health professionals perceive the coordination between the health care levels and the factors influencing it. A qualitative study was carried out based on a content analysis including 54 semistructured interviews and 11 focus groups with people from two health care networks in Xalapa and Veracruz, Mexico. The respondents reported difficulties in the follow-up to patients, discrepancies regarding the benchmark criteria, barriers in the access to specialized health care, poor quality in the reference formats, a poor reception of counterreference formats, and difficulties for the follow-up of the reference circuits. These problems were attributed to the resource insufficiency, a limited coverage by the Social Security System, work overload, poor supervision, poorly educated general practitioners, and a limited communication between the health professionals. These results agree with other studies conducted in this region. Therefore, there is a need to strengthen the mechanisms based on the normalization, and also to introduce strategies based on the mutual adaptation and modify the characteristics of the organizational structure and culture that in order to manage to coordinate an effective health care and contribute to the network integration.

Keywords: health systems, health services, quality of health care, continuity in the care to patient, qualitative research.

\section{Resumo}

A limitada coordenação assistencial impede garantir um atendimento integrado, eficiente e de qualidade. O objetivo do estudo foi explorar a percepção de profissionais de saúde sobre a coordenação entre níveis de atenção e os fatores que a influenciam. Foi realizado um estudo qualitativo mediante análise de conteúdo de 54 entrevistas semiestruturadas e 11 grupos focais com pessoal de duas redes de serviços de saúde em Xalapa e Veracruz, México. Os entrevistados relataram dificuldades no acompanhamento dos pacientes, discordâncias quanto aos critérios de referência, barreiras ao acesso à assistência especializada, baixa qualidade dos formatos de referência, escassa recepção dos formatos de contra-referência e dificuldades para acompanhar os circuitos de referência. Atribuíram esses problemas à insuficiência de recursos, a cobertura limitada do Seguro Popular, a sobrecarga de trabalho, falta de supervisão, treinamento deficiente dos clínicos gerais e comunicação limitada entre os profissionais. Os resultados coincidem com outros estudos na região, pelo que se indica a necessidade de fortalecer os mecanismos baseados na padronização, introduzir estratégias baseadas na adaptação mútua e modificar características da estrutura e cultura organizacional para alcançar uma coordenação da atenção efetiva, o que contribui à integração das redes.

Palavras-chave: sistemas de saúde, serviços de saúde, qualidade da assistência de saúde, continuidade da assistência ao paciente, pesquisa qualitativa. 


\section{Introducción}

Los sistemas de salud en América Latina se caracterizan por su fragmentación y su deficiente coordinación, lo que produce una gestión ineficiente y afecta la continuidad de la atención, considerada como una dimensión central de la calidad (1). Esto es más evidente en pacientes con enfermedades crónicas que requieren atención de múltiples profesionales de la salud y en diferentes lugares a lo largo del tiempo $(2,3)$.

Para afrontar esta problemática, los países miembros de la Organización Panamericana de la Salud (OPS) adoptaron una resolución en el año 2009 para implementar Redes Integradas de Servicios de Salud (RISS), como estrategia de gestión orientada a superar la fragmentación de la atención y, con ello, superar problemas de acceso, calidad y eficiencia que han aquejado a los sistemas de salud de la región, permitiéndoles responder mejor a las necesidades y expectativas de las personas, las familias y las comunidades (4).

No obstante, esta política pública ha encontrado dificultades en su implementación (5), y la evidencia sobre su impacto no es suficiente. Este vacío dio lugar al proyecto de investigación "Impacto de las estrategias de integración de la atención en el desempeño de las redes de servicios de salud en América Latina (Equity-LA II)", desarrollado en ocho instituciones de educación superior y de salud en América Latina, con el propósito de evaluar la efectividad de diversas estrategias de integración de la atención en la mejora de la coordinación y la calidad de la atención en las RISS en distintos sistemas de salud de la región, para desarrollar propuestas sobre buenas prácticas de integración de la atención. Dicho proyecto comprendió cuatro etapas: un estudio de línea de base, el diseño e implementación de intervenciones, la evaluación de las intervenciones y un análisis comparativo transnacional (6). Este artículo presenta resultados del estudio de línea base en donde se exploraron las percepciones que sobre la coordinación asistencial tienen los profesionales de salud de dos redes que atienden a población sin seguridad social en Veracruz, México.

En el caso de nuestro país, la principal fragmentación resulta de las instituciones creadas para atender a la población que goza de seguridad social por su condición laboral y la que no tiene acceso a esa condición, donde además existen problemas de coordinación entre esas redes y al interior de cada una. La población que no goza de seguridad social, cuya atención es brindada por las unidades médicas y el personal de salud que forman parte del presente estudio, es atendida a través de la afiliación voluntaria al Sistema de Protección Social en Salud, el cual financia un paquete limitado de servicios que es coordinado en cada una de las 32 entidades federativas por los Regímenes Estatales de Protección Social Salud (REPSS). Estos, a su vez, compran los servicios a las Secretarías de Salud Estatales, a otras instituciones de seguridad social o a entes privados, con la finalidad de proveer a la población de la atención requerida, siempre y cuando se encuentre cubierta por su catálogo de servicios. Este modelo de financiamiento, basado en los eventos de enfermedad, no ha garantizado la mejora en las condiciones de salud poblacionales y el acceso a los servicios de salud con base en la necesidad de la persona $(7,8)$. 
Angélica Ivonne Cisneros Luján / Dulce María Cinta Loaiza /

María Alejandra Sánchez Bandala / Vianey González Rojas

La actual administración, que inició funciones en 2018, ha propuesto el nuevo modelo de salud para el bienestar, que contempla la provisión de servicios de salud y medicamentos gratuitos de acuerdo con las necesidad de la persona, la familia y la comunidad, a través de un esquema que concentra las funciones de rectoría, prestación de servicios y financiamiento en la Secretaría de Salud Federal, de manera centralizada (9).

\section{Marco conceptual}

Todo sistema de salud cuenta con un modelo de atención que define la organización de los diferentes niveles de prestación de servicios, los recursos necesarios para su funcionamiento y el flujo de pacientes entre ellos. Esta organización puede tener un diseño de red, que se considera integrada cuando la oferta de servicios responde a las características de la población a cargo, tiene un sistema de gobernanza único y participativo, se reconoce al primer nivel como la entrada al sistema y el responsable de coordinar el cuidado del paciente, y cuenta con un sistema de información compartido (10). La coordinación, entendida como una relación estructurada entre servicios o niveles organizativo (1), se considera una condición necesaria para lograr la integralidad de las redes (11).

Así, las RISS son un esquema de prestación de servicios de salud que se define como "el conjunto de organizaciones que ofrecen una atención coordinada a través de un continuo de prestaciones a una población determinada, y que se responsabilizan de los costes y resultados en salud de la población" (12). En tanto que la coordinación asistencial se refiere a "la concertación de todos los servicios relacionados con la atención del paciente, con independencia del lugar donde se reciban, de manera que se armonicen y se alcance un objetivo común, administrando los posibles conflictos" (6).

De acuerdo con la literatura, la coordinación puede clasificarse en tres tipos (13). La coordinación de la gestión clínica comprende la provisión de atención de manera secuencial y complementaria, dentro de un plan de atención compartido por los diferentes niveles de atención y servicios que participan. Este tipo de coordinación se define a partir de tres dimensiones: la coherencia de la atención (aproximaciones y objetivos de tratamiento similares), la accesibilidad entre niveles y el seguimiento adecuado del paciente en sus transiciones entre niveles de atención (6). La coordinación de la información se refiere a la transferencia y uso de información sobre episodios anteriores de enfermedad y situación biopsicosocial del paciente (14). La coordinación administrativa se refiere a la coordinación del acceso del paciente a lo largo del continuo asistencial de acuerdo con sus necesidades (6). Como factores que inciden en la coordinación se mencionan los correspondientes al sistema de salud, a la red, a los profesionales y a los usuarios.

Para favorecer la coordinación pueden utilizarse distintos mecanismos, algunos se basan en una normalización de las habilidades (formación continua) o de los procesos de trabajo (Guías de Práctica Clínica - GPC-, protocolos y circuitos para la referencia de pacientes), en tanto que otros se basan en la comunicación directa entre actores que intervienen en 
Percepción sobre la coordinación de la atención: el caso de las redes de servicios de salud de Xalapa y Veracruz, México, en el periodo 2014-2016

un proceso, permitiendo una adaptación mutua (comunicación informal, grupos de trabajo multidisciplinarios, puestos de enlace, sistemas de información vertical) (15) (Tabla 1).

Tabla 1. Categorías y dimensiones de la coordinación de la atención

\begin{tabular}{|c|c|}
\hline Categorias & Dimensiones \\
\hline \multirow[t]{3}{*}{ Coordinación de gestión clinica } & Seguimiento adecuado del paciente \\
\hline & Accesibilidad entre niveles \\
\hline & Coherencia de la atención \\
\hline \multirow{2}{*}{$\begin{array}{l}\text { Coordinación } \\
\text { de información }\end{array}$} & $\begin{array}{l}\text { Transferencia de la información clinica y } \\
\text { biopsicosocial }\end{array}$ \\
\hline & Utilización de la información \\
\hline \multirow[t]{2}{*}{ Coordinación administrativa } & Circuitos administrativos establecidos \\
\hline & Ordenación del acceso \\
\hline \multirow[t]{4}{*}{ Factores de la coordinación } & Factores del sistema de salud \\
\hline & Factores de la red \\
\hline & Factores relacionados con los profesionales \\
\hline & Factores relacionados con los usuarios \\
\hline \multirow[t]{3}{*}{ Propuestas } & Propuestas sobre el sistema de salud \\
\hline & Propuestas sobre la organización \\
\hline & Propuestas sobre los profesionales \\
\hline \multirow[t]{2}{*}{ Mecanismos de coordinación } & Normalización (habilidades/procesos de trabajo) \\
\hline & $\begin{array}{l}\text { Adaptación mutua (comunicación informal, sistemas } \\
\text { de información vertical, dispositivos de enlace) }\end{array}$ \\
\hline
\end{tabular}

Fuente: elaboración propia con base en Vázquez et al. (15) 
Angélica Ivonne Cisneros Luján / Dulce María Cinta Loaiza /

María Alejandra Sánchez Bandala / Vianey González Rojas

Algunos de estos mecanismos han sido concebidos para su implementación al interior de las organizaciones de salud, como los sistemas de información vertical para compartir información entre unidades de diferentes niveles asistenciales, o los sistemas de referencia y contrarreferencia que, además de transmitir información, comprenden procesos clínicos y administrativos que organizan el tránsito de pacientes por servicios con distinta capacidad resolutiva, para atender sus necesidades en salud. Sin embargo, estos mecanismos se han adaptado para su funcionamiento en el ámbito de las RISS, dado que estas suelen comprender unidades organizadas en niveles de atención en donde se desarrollan distintas etapas del proceso asistencial.

\section{Método}

Dado el carácter internacional del proyecto Equity-LA II, el diseño metodológico fue construido de manera colectiva por los investigadores de los países participantes, tomando como referencia los resultados de un proyecto antecedente. Sin embargo, dada la diversidad y complejidad de los sistemas de salud que abarca el proyecto y sus respectivos contextos, se acordó realizar los ajustes y adaptaciones necesarias a los instrumentos a usar en cada país.

Para el caso de México, como parte de la primera etapa se desarrolló un estudio cualitativo sustentado en entrevistas individuales semiestructuradas y grupos focales con personal de salud que atiende a población urbana, con padecimientos crónicos con énfasis en diabetes mellitus tipo 2, enfermedad pulmonar obstructiva crónica (EPOC) e hipertensión arterial (HTA).

Los países participantes de Equity-LA II manejaron como criterios comunes para la selección de sus redes de estudio que en ellas se atendiera a una población definida, urbana y de nivel socioeconómico medio o medio-bajo, que en dichas redes se brindara un continuo de servicios de al menos atención primaria y especializada, y que sus autoridades manifestaran interés por participar en el estudio. Para el caso de México se decidió trabajar con dos redes que atienden a un volumen similar de población sin seguridad social en los dos municipios más poblados del estado de Veracruz: Xalapa, con 458.000 habitantes, de los cuales 225.326 no gozan de seguridad social, y Veracruz, con 552.000 habitantes, de los cuales 292.274 no gozan de seguridad social (16). Se excluyeron del estudio a las unidades que no atienden pacientes con las enfermedades que fueron consideradas como trazadoras (17), por lo que las redes quedaron conformadas para fines del estudio, como se muestra en la Tabla 2. 
Percepción sobre la coordinación de la atención: el caso de las redes de servicios de salud de Xalapa y Veracruz, México, en el periodo 2014-2016

Tabla 2. Unidades por nivel de complejidad que conforman el área de estudio en las dos redes seleccionadas

\begin{tabular}{|c|c|c|}
\hline $\begin{array}{l}\text { Nivel de } \\
\text { complejidad }\end{array}$ & Red Xalapa & Red Veracruz \\
\hline I nivel & $\begin{array}{l}\text { 1. CS. Col. Arroyo Blanco } \\
\text { 2. CS. Col. Emiliano Zapata } \\
\text { 3. CS. Col. Revolución } \\
\text { 4. CS. Dr. Gastón Melo } \\
\text { 5. CS. Dr. José A. Maraboto } \\
\text { 6. CS. Lerdo de Tejada } \\
\text { 7. CS. Miguel Alemán }\end{array}$ & $\begin{array}{l}\text { 1. CS. } 21 \text { de Abril } \\
\text { 2. CS. Anastasio Iturralde } \\
\text { 3. CS. Club de Leones } \\
\text { 4. CS. Delfino Victoria } \\
\text { 5. CS. El Coyol } \\
\text { 6. CS. Fracc. Los Pinos } \\
\text { 7. CS. Granja Rio Medio } \\
\text { 8. CS. Las Amapolas } \\
\text { 9. CS. Las Bajadas } \\
\text { 10. CS. Mata Cocuite } \\
\text { 11. CS. Reserva Tarimoya } \\
\text { 12. CS. Ruiz Cortines } \\
\text { 13. CS. Valente Diaz } \\
\text { 14. CS. Vargas } \\
\text { 15. CS. Virgilio Uribe }\end{array}$ \\
\hline $\begin{array}{l}\text { Unidades } \\
\text { intermedias }\end{array}$ & $\begin{array}{l}\text { 1. Unidad de Especialidades Médicas } \\
\text { de Enfermedades Crónicas (Uneme- } \\
\text { EC) }\end{array}$ & \\
\hline $\begin{array}{l}\text { II nivel y III } \\
\text { nivel }\end{array}$ & $\begin{array}{l}\text { 2. Hospital Regional de Xalapa "Dr. Luis } \\
\text { F. Nachón" } \\
\text { 3. Centro de Alta Especialidad "Dr. } \\
\text { Rafael Lucio" }\end{array}$ & $\begin{array}{l}\text { 1. Hospital General de } \\
\text { Tarimoya "Dr. Horacio } \\
\text { Diaz Cházaro" } \\
\text { 2. Hospital de Alta } \\
\text { Especialidad de Veracruz }\end{array}$ \\
\hline
\end{tabular}

Fuente: elaboración propia con información del Catálogo de Clave Única de Establecimientos de Salud (Clues) (17).

En las dos redes fueron seleccionados profesionales de salud de los diferentes niveles de atención directamente involucrados en el proceso de coordinación de la atención, con una antigüedad en la función desempeñada de al menos 6 meses, y que aceptaran participar en la investigación. Se definieron cuatro grupos: 1) profesionales de primer nivel: médicos generales y equipos zonales de supervisión; 2) profesionales de segundo y tercer nivel: médicos especialistas de urgencias, consulta externa, medicina interna y neumología, involucrados en la atención de las patologías crónicas trazadoras; 3) mandos medios: director de centro de salud de primer nivel, responsable de referencia y contrarreferencia de las redes y a nivel estatal, director y subdirector de hospital; y 4) personal administrativo y paramédico: trabajadoras sociales, enfermeras, personal de laboratorio clínico, gestor médico del Seguro Popular (mecanismo de aseguramiento del Sistema de Protección Social en Salud) y auxiliares administrativos de control de citas médicas. 


\section{Angélica Ivonne Cisneros Luján / Dulce María Cinta Loaiza /}

María Alejandra Sánchez Bandala / Vianey González Rojas

El tamaño de la muestra se definió por el criterio de saturación, el cual se alcanza cuando la información recopilada no aporta nada nuevo a la exploración de las propiedades y dimensiones de las categorías de análisis. Con estos actores se llevaron a cabo 54 entrevistas individuales semiestructuradas y 11 grupos focales, en los cuales participaron entre 7 y 11 profesionales. La composición de la muestra se presenta en la Tabla 3. El instrumento utilizado fue una guía de grupo focal/entrevista individual sobre coordinación de la atención, que contempló una ficha de identificación con el nombre del participante, su edad, sexo, titulación, cargo, institución y tiempo en el cargo, la hora de inicio y finalización de la entrevista y el nombre de los moderadores. La guía estuvo compuesta por tres ejes que fueron: 1) la coordinación de la atención entre niveles en la red; 2) los mecanismos de coordinación entre niveles de atención; y 3 ) el concepto de coordinación asistencial.

Tabla 3. Composición de la muestra de informantes para el análisis de la coordinación de la atención entre niveles

\begin{tabular}{|c|c|c|c|}
\hline & & Red Xalapa & Red Veracruz \\
\hline \multirow{5}{*}{$\begin{array}{l}\text { Entrevistas } \\
\text { individuales }\end{array}$} & Profesionales de I nivel & 9 & 6 \\
\hline & Profesionales de II y III nivel & 5 & 6 \\
\hline & Mandos medios/gerentes/directivos & 6 & 7 \\
\hline & $\begin{array}{l}\text { Personal administrativo y } \\
\text { paramédico }\end{array}$ & 8 & 7 \\
\hline & Total & 28 & 26 \\
\hline \multirow{5}{*}{$\begin{array}{l}\text { Grupos } \\
\text { focales }\end{array}$} & Profesionales de I nivel & 2 & 2 \\
\hline & Profesionales de II y III nivel & 0 & 2 \\
\hline & $\begin{array}{l}\text { Grupos mixtos (profesionales de I, II y } \\
\text { III nivel y personal de Jurisdicción } \\
\text { Sanitaria) }\end{array}$ & 1 & 1 \\
\hline & Grupos de discusión & 1 & 2 \\
\hline & Total & 4 & 7 \\
\hline
\end{tabular}

Fuente: elaboración propia.

Atendiendo a los requerimientos éticos de la investigación, en todos los casos se obtuvo por escrito el consentimiento informado de los entrevistados y de los participantes de los grupos focales. La recolección de la información se llevó a cabo entre mayo del 2014 y noviembre del 2015. Las entrevistas y los grupos focales se grabaron en audio con consentimiento de los participantes, se transcribieron, categorizaron y se realizó un análisis temático de contenido con apoyo del programa Atlas-ti. Las categorías de análisis, basadas en el marco conceptual del proyecto, se muestran en la Tabla 1. 
En enero y febrero de 2016, de acuerdo con lo establecido en las directrices de la investigación, se llevaron a cabo sesiones de devolución de resultados en los centros de salud en los que se trabajó, lo que permitió precisar y profundizar en algunos de los hallazgos.

\section{Resultados}

En este apartado se presentan los resultados del estudio con un alcance descriptivo, para profundizar en su discusión e interpretación en el siguiente apartado.

En ambas redes de estudio se identificaron problemas de coordinación de la atención entre niveles muy similares. Un primer hallazgo fue que los profesionales entrevistados asimilan el concepto de coordinación al sistema de referencia y contrarrefencia — que la literatura reconoce como uno de los mecanismos que favorecen la coordinación de la atención-, desconociendo a otros mecanismos como parte de los esfuerzos para lograr una atención coordinada, a pesar de que participen de ellos en cierta medida en su entorno laboral.

\section{Coordinación de gestión clínica}

Respecto al seguimiento, los entrevistados coincidieron en señalar al médico general o al personal del centro de salud como responsables de esta función, sin embargo, mencionaron que esta no se cumple de manera adecuada. En opinión de los profesionales de primer nivel esto se debe a que los pacientes no regresan al centro de salud o lo hacen sin la contrarreferencia, debido a que el paciente (que es quien, en la práctica, se encarga de llevar el documento) la perdió, o porque el médico especialista no la hizo, lo cual reconocieron algunos médicos de segundo nivel. Señalan, además, que esta función se les dificulta por la sobrecarga de trabajo, lo cual fue mencionado también por los directivos.

Para hacer el seguimiento de los pacientes que no regresan a primer nivel, los entrevistados señalaron la existencia de visitas domiciliarias a cargo del personal del centro de salud, quienes reconocieron que no siempre se realizan debido a falta de personal para realizarlas; que los pacientes no suelen informar de cambios en su domicilio; y que no existen recursos destinados a esta actividad:

A nosotros no nos dan un apoyo económico en cuestión de viáticos para salir a hacer visita domiciliaria, entonces buscamos la primera opción que es vía telefónica...que tampoco recibimos un apoyo económico, lo hacemos directamente del celular nuestro (Personal administrativo 01 de primer nivel de atención, red Xalapa).

Los entrevistados en cargos administrativos compartieron esta opinión, no así los médicos especialistas, quienes atribuyeron la falta de visitas domiciliaras a problemas de actitud del personal del primer nivel: 
Angélica Ivonne Cisneros Luján / Dulce María Cinta Loaiza /

María Alejandra Sánchez Bandala / Vianey González Rojas

[...] es muy regular que le toca a la enfermera ir de tal manzana, localizar tal paciente... y simplemente dice "no lo encontré" aunque no lo busque [...] No se hace porque "no tengo tiempo", "no quiero" (Profesional de salud 01 de segundo nivel de atención, grupo focal 04, red Veracruz).

En cuanto a la coherencia de la atención, algunos entrevistados señalaron inconsistencias entre los niveles de atención respecto al diagnóstico y tratamiento, lo que relacionaron con deficiencias en la formación de los médicos generales o con las diferentes fuentes de información y referencia utilizadas por los profesionales:

Veíamos un caso clínico y en un primer nivel todos estamos de acuerdo con el diagnóstico y tratamiento y segundo nivel ¡No! Nada que ver, entonces existe una gran diferencia que no debería de ser porque la norma es igual para todos, sin embargo, ellos ya manejan otros tipos de esquemas, otros tipos de tratamientos, que del artículo fulano de tal [...] (Personal directivo 04 de primer nivel de atención, red Xalapa).

Los entrevistados coincidieron en señalar que los médicos generales suelen hacer referencias a segundo nivel innecesarias, incompletas, a la especialidad inadecuada o sin estudios que respalden el envío, cuestión que incluso los médicos de primer nivel reconocieron como un problema. Los médicos especialistas - y algunos directivos y administrativos- afirmaron que esto se debe a la deficiente formación de los médicos generales y a falta de interés en realizar su trabajo. Los médicos generales, aunque reconocieron que necesitan capacitarse, señalaron que en ocasiones realizan referencias que no se justifican clínicamente debido a la falta de insumos en los centros de salud para la atención del paciente, o ante la imposibilidad de resurtir medicamentos que solo pueden otorgarse en segundo nivel. Además, destacaron que algunas de las referencias que realizan en apego a las normativas (Normas Oficiales Mexicanas y GPC) son rechazadas de manera injustificada en segundo nivel, debido a que los especialistas y el personal de enlace no se apegan a dichos lineamientos:

Cuando hacemos esta referencia, que es normativa, hemos tenido muchísimas dificultades [...] porque consideran segundo y tercer nivel que ese servicio, esa acción, no les corresponde [...] ya nos aceptan al paciente cuando ya presenta una complicación [...], pero enviar una referencia de manera normativa, preventiva, no nos lo están aceptando (Personal directivo 03 de primer nivel de atención, red Xalapa).

Estas inconsistencias en cuanto al momento de referir a un paciente a otro nivel también se evidenciaron respecto al regreso de pacientes al centro de salud, pues algunos médicos especialistas refirieron retener pacientes con enfermedades crónicas como subsecuentes en la atención especializada — sin notificar al primer nivel— debido al riesgo de exacerbación, o porque necesitan ser tratados por un grupo multidisciplinario:

Los diabéticos que me llegan ya no los doy de alta, ya se quedan conmigo para siempre [...] deben quedarse en el hospital para que sean tratados por el grupo multidisciplinario (Profesional de salud 01 de segundo nivel de atención, grupo focal 06, red Xalapa). 
En cuanto a las pruebas diagnósticas que se indican a los pacientes, médicos generales y directivos consideraron que en el segundo nivel se tiende a duplicar las pruebas, sin embargo, entre los médicos especialistas prevaleció la opinión de que las pruebas solo se repiten cuando es necesario:

¿Duplicarlos? Solo tal vez cuando sean necesarios, porque [...] a lo mejor el paciente me trae un examen cuando el médico lo mandó referido con nosotros, pero esa referencia, ese examen se lo hizo tres meses antes, tal vez ese examen ya no me sea útil (Profesional de salud 04 de segundo nivel de atención, red Xalapa).

Estas diferencias de criterios entre profesionales de diferentes niveles de atención contribuyen a un clima de desconfianza entre y a comentarios despectivos de los especialistas respecto a las capacidades clínicas de los médicos generales.

En lo concerniente al acceso entre niveles, todos los actores valoraron de manera positiva el acceso a urgencias, y los médicos de ambos niveles destacaron que el Seguro Popular ha facilitado el acceso a la atención médica. Además, personal de primer nivel y directivos destacaron la utilidad de mecanismos de comunicación informal como celulares, teléfono o correo electrónico para facilitar el acceso de algunos pacientes al segundo nivel, aunque su uso no es extendido.

Sin embargo, los diferentes actores coincidieron en señalar barreras de acceso al segundo o tercer nivel de atención, a estudios de laboratorio y a algunos medicamentos; así como el diferimiento prolongado para citas de consulta externa especializada:

El diferimiento de citas es muy grande. Entonces, si nosotros queremos una valoración de neumología, la próxima oportunidad es en tres, cuatro o cinco meses (Personal directivo 06 de segundo nivel de atención, red Veracruz).

Los entrevistados atribuyeron estos problemas a la saturación de los servicios hospitalarios que, a su vez, relacionaron con los limitados recursos materiales y humanos o la deficiente gestión de estos, problemas exacerbados —en su opinión — desde la entrada en vigor del Seguro Popular, que aumentó la demanda de atención sin proveer los recursos necesarios. Coincidieron también en señalar que la cobertura limitada de este seguro actúa como otra barrera para el acceso de los pacientes a consultas, estudios y tratamientos sin costo:

Solamente creo que aceptan uno o dos estudios por enfermedad, si el paciente requiriera otro estudio ya no lo aceptan, el paciente lo tiene que pagar [...] el Seguro Popular no se los cubre (Personal administrativo 04 de primer nivel de atención, red Xalapa).

Como consecuencias de estas barreras de acceso, los entrevistados mencionaron el gasto de bolsillo que los pacientes deben realizar, la interrupción del tratamiento, el deterioro de su cuadro clínico, o la insatisfacción de los usuarios. 
Angélica Ivonne Cisneros Luján / Dulce María Cinta Loaiza /

María Alejandra Sánchez Bandala / Vianey González Rojas

\section{Coordinación de información}

Respecto a la transferencia de información, los entrevistados identificaron al formato de referencia y contrarreferencia como el principal mecanismo de coordinación utilizado. Sobre este, varios médicos especialistas señalaron que los médicos generales lo elaboran con letra ilegible y la información que contiene es incompleta e incongruente.

Hay referencias que vienen con... pues con datos que les faltan, pues no cumplen con la norma del expediente clínico y traen datos incompletos (Personal directivo 07, red Veracruz).

Respecto al formato de contrarreferencia, los médicos generales señalaron que su recepción es escasa y tardía. Estos resultados son congruentes con la percepción del personal administrativo y directivo, quienes mencionaron que son pocas las contrarreferencias recibidas en primer nivel. Los médicos generales, directivos y administrativos identificaron que esta falta de recepción de contrarreferencias se debe a que el paciente no entrega el formato, pues desconoce su función, $o$ a que los especialistas no las elaboran debido a la sobrecarga de trabajo en el nivel hospitalario, y a que no se les supervisa esta actividad:

La permisividad que se les ha dado a ellos [los especialistas] de no hacer lo que no quieren hacer [contrarreferencias], yo creo que es disciplina y no todo el mundo es disciplinado (Personal administrativo 08, red Xalapa).

Al no recibir la contrarreferencia, los médicos generales indicaron que se ven limitados para llevar a cabo el seguimiento de los pacientes y para brindarles una atención de calidad, ya que deben recurrir a las recetas, los carnés de citas y la información que brindan los propios pacientes para conocer los procedimientos realizados en el segundo nivel. Directivos y administrativos indicaron que, efectivamente, el médico general debe recurrir a estos medios informales para conocer lo sucedido en la atención especializada:

Desafortunadamente no siempre recibimos la contrarreferencia, muchas veces la información la tenemos del paciente que regresa y ya nos dice qué fue lo que ocurrió en el segundo nivel (Profesional de salud 10 de primer nivel de atención, red Xalapa).

En cuanto a la información transferida entre niveles, los médicos generales, el personal directivo e incluso los médicos especialistas mencionaron que no siempre se utiliza para la toma de decisiones. Los especialistas refirieron no tomarla en cuenta debido a su baja calidad, que implica reinterrogar y explorar nuevamente al paciente:

Hay veces que la letra (de la referencia) es legible y entendible [...], pero hay otros que no se les entiende nada, y entonces pues nosotros reinterrogamos al enfermo y ya nos guiamos por eso (Profesional de salud 01 de segundo nivel de atención, grupo focal 06, red Xalapa). 


\section{Coordinación administrativa}

En ambas redes se identificaron mecanismos dirigidos a fortalecer la coordinación administrativa, sin embargo, estos presentan problemas en su aplicación. Los entrevistados coincidieron en señalar que el sistema de referencia y contrarreferencia no funciona adecuadamente, ya que los usuarios suelen acudir directamente al servicio de urgencias en el hospital, sin recurrir al centro de salud como puerta de entrada al sistema:

No se están llevando a cabo las referencias de los centros de salud. Llegan los pacientes prácticamente solos al departamento de urgencias (Profesional de salud 08 de primer nivel de atención, grupo focal 06, red Xalapa).

Además, el personal administrativo de ambas redes mencionó que algunos médicos generales refieren a unidades que no les corresponden debido a que no tienen información sobre los servicios disponibles en cada hospital. Por su parte, algunos médicos generales se manifestaron inconformes con el sistema de referencia, debido a que en ocasiones deben referir a pacientes a un hospital que les corresponde por norma, aunque sepan que este no cuenta con los servicios requeridos:

Ni siquiera lo podían recibir en el segundo nivel de Boca del Río, porque no pertenece, por regionalización le tocaba Acayucan o Luta, que ahí se les mueren peor que en ningún lado (Profesional de salud 06 de segundo nivel, grupo focal 5, red Veracruz).

En relación con esto, médicos especialistas y generales coincidieron en señalar que en algunos casos la regionalización operativa establecida puede dificultar el acceso a los servicios. Esto indica, en términos de flujos comunicacionales, una grave descoordinación administrativa.

Otro mecanismo que de acuerdo con la literatura puede favorecer la coordinación al permitir a los profesionales de ambos niveles exponer sus problemas y llegar a acuerdos son las reuniones de referencia y contrarreferencia. Sin embargo, los médicos generales y personal administrativo de la red Xalapa, así como los médicos especialistas de la red Veracruz, destacaron que estas reuniones no cumplen su cometido debido a que no participa personal operativo ni directivos con capacidad resolutiva, y a que no se socializan los acuerdos tomados en dichas reuniones, ni se les da seguimiento, es decir, estas reuniones son para altos mandos directivos, los cuales no siempre están conscientes de los problemas de ejecución administrativa cotidiana.

Otros mecanismos de coordinación mencionados por los participantes del estudio fueron los puestos de enlace, profesionales que se ocupan de gestionar el acceso a segundo nivel de los pacientes y que, desde la perspectiva de los profesionales entrevistados, funcionan como "filtro" de las referencias que llegan a los hospitales. Estos filtros son considerados por los especialistas como mecanismos útiles para contener la saturación de los centros de atención. No obstante, los médicos generales, personal directivo y administrativo los perciben como barreras de acceso a la atención especializada: 
Angélica Ivonne Cisneros Luján / Dulce María Cinta Loaiza /

María Alejandra Sánchez Bandala / Vianey González Rojas

El CEM es un hospital muy saturado, tiene un sistema de filtros y nos rechazaba los pacientes [...] de que no correspondía el envío, que podía llevar su control en el centro de salud, aunque nosotros solicitáramos una valoración endocrinológica, y sobre todo para ajustar medicamento (Profesional de salud 03 de primer nivel de atención, red Xalapa).

Frente a las problemáticas señaladas, las propuestas para mejorar la coordinación mencionadas por los entrevistados se centraron en dotar de mayores recursos - humanos y materiales - a las redes y mejorar su gestión, implementar mecanismos de supervisión a los médicos especialistas para la elaboración de contrarreferencias, replantear o flexibilizar algunos aspectos de los circuitos administrativos, introducir el expediente electrónico, implementar programas de capacitación para los médicos generales, fortalecer y reorientar las reuniones de referencia y contrarreferencia, informar a la población sobre el funcionamiento del sistema de referencia y contrarreferencia, los circuitos administrativos y la comunicación directa entre profesionales de salud.

\section{Discusión}

Los hallazgos de este estudio coinciden con investigaciones en el ámbito internacional, algunas de ellas realizadas en el marco del proyecto Equity LA-II $(11,18,19,20,21)$, en las que se evidencian importantes problemas de coordinación en los sistemas de salud de la región.

La identificación del sistema de referencia y contrarreferencia como el central o único mecanismo para la coordinación denota una insuficiente comprensión por parte de los profesionales de las redes estudiadas del concepto de coordinación de la atención, lo que coincide con los resultados de otros países participantes del proyecto internacional (11).

Esto no implica que en las redes analizadas no existan otros mecanismos de coordinación, pues fue posible identificar grupos de trabajo multidisciplinario y puestos de enlace. Sin embargo, los profesionales no los reconocen como orientados a mejorar la coordinación, sino que los asumen como reuniones de evaluación o filtros para las referencias enviadas a segundo nivel.

Esto indica que la propuesta conceptual de la coordinación de la atención que se maneja en la literatura especializada y en la política pública ha permeado escasamente el quehacer cotidiano de los profesionales de salud de la región, dando lugar a lo señalado por otros estudios como una "brecha de información" entre los encargados del diseño de la política y los responsables de implementarla (22). Esto implica la necesidad de capacitación al respecto y un área de oportunidad para generar intervenciones basadas en mecanismos de coordinación no utilizados $(23,24)$.

En cuanto a la coordinación de la gestión clínica, los profesionales de las redes analizadas reconocieron al primer nivel como el responsable del seguimiento de pacientes a lo largo del continuo asistencial, lo que representa un avance respecto a la adopción de un modelo de 
redes integradas basadas en el primer nivel de atención. Sin embargo, los pacientes parecen no reconocer esta función, pues muchos de ellos no regresan a sus centros de salud después de ser contrarreferidos, y los médicos especialistas no colaboran con un seguimiento adecuado al no cumplir con el envío del formato de contrarreferencia.

Al igual que en otros estudios $(20,25,26,27)$, se identificaron dificultades para el seguimiento de los pacientes y discrepancias entre médicos generales y especialistas sobre tratamientos y criterios de derivación, lo que da lugar a referencias innecesarias, y contribuye a la saturación de los hospitales y a aumentar el diferimiento de citas de especialidad. Este fenómeno, tal como lo señalan otros autores $(28,29)$, puede empeorar la situación de salud y aumentar los costos de atención, que también se incrementan cuando pacientes con padecimientos crónicos controlados son retenidos innecesariamente en el segundo nivel, lo cual ha sido reportado en otros estudios a nivel nacional (30).

Respecto a la coordinación de información, similar a lo reportado para Chile, se identificó que el envío del formato de referencia se realiza, posiblemente, porque para ambos países es requisito para la derivación de pacientes al segundo nivel (19). Sin embargo, coincidiendo con lo reportado por otros estudios $(11,31,32)$, los médicos especialistas consideraron que las referencias que reciben son inadecuadas y de baja calidad, por lo que no son tomadas en cuenta para la toma de decisiones clínicas (33). Este uso del formato de referencia con fines administrativos más que de intercambio de información fue identificado también en Colombia (19).

La limitada recepción de contrarreferencias en primer nivel es otro de los problemas identificados en la literatura nacional e internacional $(30,34,35)$, lo que lleva a adjudicar a los usuarios una función que no les corresponde como transmisores de la información en su transición de la atención especializada a la atención primaria $(36,37)$.

Para afrontar estas dificultades en la transferencia y uso de información, algunos países desarrollados han incorporado tecnologías de la información y comunicación (TIC), tales como el expediente clínico electrónico. Sin embargo, en las redes analizadas, como en el resto de los países participantes del proyecto internacional, no se identificaron mecanismos basados en las TIC (20). Es tarea pendiente valorar la viabilidad en el mediano plazo de implementar estos mecanismos en los países de la región, no obstante, será conveniente atender la sugerencia de Llanusa et al. respecto a no idealizar las ventajas de estos sistemas y mantener una aproximación equilibrada que destaque el papel fundamental de los recursos humanos en el logro de resultados efectivos en salud (38).

En cuanto a la coordinación administrativa, los hallazgos coinciden con investigaciones previas al identificar una demanda innecesaria en los servicios de urgencias $(15,16,39)$ y limitaciones del circuito administrativo para atender las necesidades de los usuarios, lo cual puede indicar una falta de visión centrada en la persona en las redes estudiadas (40). 
Angélica Ivonne Cisneros Luján / Dulce María Cinta Loaiza /

María Alejandra Sánchez Bandala / Vianey González Rojas

Los factores a los que se atribuyen los problemas de coordinación coinciden, en lo general, con los identificados en otros estudios que destacan la insuficiencia de recursos humanos y físicos o deficiente gestión de estos, lo que deriva en altas cargas de trabajo $(18,25,26,41,42)$, la comunicación inadecuada entre médicos generales y especialistas $(40,43)$, la desconfianza de los médicos especialistas respecto a las capacidades y actitudes de los médicos generales (19), la existencia de problemas actitudinales y de descalificación entre profesionales (27), y la falta de información y formación en la población para una utilización adecuada de los servicios de salud $(5,43)$.

Además, los profesionales hicieron referencia al modelo de aseguramiento conocido como Seguro Popular como otro de los factores que impactan en la coordinación de la atención, al aumentar la demanda de atención sin incrementar los recursos entregados, y restringir el acceso a medicamentos y estudios, debido a que ofrece un paquete limitado de prestaciones a sus afiliados. Resultados similares se han reportado en otros estudios nacionales (44) y en aquellos realizados en sistemas de salud, como el colombiano, donde se ha desarrollado un modelo de competencia gestionada (18).

Tal como lo señalan estudios previos, los profesionales entrevistados mencionaron que los problemas de coordinación disminuyen la eficiencia de las redes, aumentan la saturación de los hospitales (28), y limitan la continuidad de la atención de los pacientes (45). Además, en este estudio se señaló también un impacto negativo en el gasto de bolsillo de los usuarios y en la interrupción de su tratamiento.

Las propuestas de los participantes de este estudio son similares a las reportadas en otras investigaciones, que apuntan a aspectos macroestructurales (planificación, recursos, modelo de aseguramiento), organizacionales (reestructuración de procesos, supervisión, condiciones laborales) (46), y relacionados con profesionales (capacitación y comunicación entre médicos de diferentes niveles) $(25,27,32)$.

Sin embargo, este estudio permitió profundizar en estos aspectos y precisar que cuando se menciona la necesidad de capacitación del personal, se piensa en términos de formación en las funciones de sus puestos, pero también en el modelo de gestión en redes integradas. Por otra parte, cuando se aborda la sobrecarga de trabajo, se destaca que es necesario revisar y modificar los procesos tradicionales para que las tareas requeridas para el funcionamiento del modelo no se sobrepongan simplemente y se perciban por el personal como una carga extra e innecesaria. Se identificó también que es indispensable fortalecer a la atención primaria en cuanto a recursos humanos, materiales y técnicos, con el fin de que pueda cumplir con eficacia su rol como puerta de entrada a las redes y como un nivel con alta capacidad resolutiva.

Así mismo, cuando se habla de mejorar la comunicación entre profesionales, se destaca la impostergable tarea de transformar una cultura organizacional donde los profesionales de 
diferentes niveles no logran verse como parte de un mismo proceso asistencial y en la que se aprecia lo que Ferreira et al. (21) llaman una "cultura de supremacía" de los especialistas, que entorpece la colaboración entre los profesionales. Además, se destaca la necesidad de informar y educar a la población para la utilización adecuada de los servicios en el marco del derecho a la salud y del modelo de gestión en redes.

\section{Conclusiones}

Los hallazgos de esta investigación permiten documentar los problemas de coordinación en las redes de estudio, que en gran medida representan una problemática nacional y regional que es urgente atener dadas sus consecuencias en la continuidad de la atención, la salud de la población, la economía de los usuarios y los costos para el sistema de salud.

La coordinación de la atención es uno de los atributos que contribuyen a la integración de las redes, y fue el objeto de estudio de este trabajo, sin embargo, al analizar sus limitaciones se evidenciaron debilidades en otros atributos de las redes estudiadas. Siguiendo a Tabarquino et al. (47), si se acepta que la aplicación efectiva del concepto de redes integradas requiere un compromiso institucional para modificar estructuras organizacionales y procesos de trabajo, los resultados de este estudio sugieren que para lograr una implementación exitosa del modelo en el contexto de estudio, será indispensable fortalecer un gobierno común a la red, capaz de orientar a los actores hacia el logro de objetivos compartidos, fortalecer y transformar el primer nivel para adecuarlo al rol que le corresponde dentro del modelo, desarrollar un sistema de información compartido, construir una cultura organizacional que favorezca la cooperación, fortalecer los mecanismos de coordinación basados en la estandarización de procesos y habilidades, y retomar la importancia de los mecanismos basados en la adaptación mutua que propicien la comunicación directa entre profesionales.

Cabe recordar que en este estudio se trabajó con servicios que atienden a población sin seguridad social, por lo que sería conveniente analizar estos procesos en poblaciones con otras formas de aseguramiento y en otras regiones del país, por lo que se recomienda realizar más investigaciones en esta línea.

La contrastación de los resultados del estudio con los de aquellos realizados en el ámbito internacional permitió observar que los problemas de coordinación, aunque similares, obedecen a procesos diferentes en función de los rasgos que definen los sistemas y políticas de salud en cada contexto. Esta información resulta útil en la actual transición del sistema de salud mexicano de un modelo basado en la competencia gestionada y aseguramiento subsidiado a un sistema público centralizado pues puede orientar un ejercicio de planeación estratégica para la aplicación efectiva de RISS. 
Angélica Ivonne Cisneros Luján / Dulce María Cinta Loaiza /

María Alejandra Sánchez Bandala / Vianey González Rojas

\section{Agradecimientos}

Agradecemos a las personas entrevistadas, a las instituciones prestadoras de servicios de salud que colaboraron en este trabajo, y especialmente a la Comisión Europea, Programa FP7, cuyo apoyo financiero fue imprescindible para la realización del estudio.

\section{Referencias}

1. Torres A, Lledó R. La coordinación entre niveles asistenciales. Rev Calidad Asistencial. 2001; 16:232-233.

2. Banco Mundial. Population aging: is Latin America ready. Washington: Cotlear Daniel; 2011.

3. McAdam M. Framework of integrated care for the elderly. Ontario: Canadian Policy Research Network; 2008.

4. Organización Panamericana de la Salud. Redes Integradas de Servicios de Salud basadas en la Atención Primaria de Salud, Resolución CD49.R22 [Internet]. Washington: OPS; 2009 [revisión 2017 jul. 3]. Disponible en: http://new.paho.org/hq/dmdocuments/2009/CD49.R22\%20(Esp.).pdf

5. Vázquez ML, Vargas L. Redes Integradas de Servicios de Salud: ¿Solución o problema? Cienc Salud. 2006;4(1):5-9.

6. Vázquez ML, Vargas I, Unger J, De Paepe P, Mogollón-Pérez A, Samico I, et al. Evaluating the effectiveness of care integration strategies in different healthcare systems in Latin America: the EQUITY-LA II quasi-experimental study protocol. BMJ Open [Internet]. 2015;5(7):e007037. Disponible en: http://bmjopen.bmj.com/content/5/7/e007037

7. Laurell AC, Ronquillo J. La Segunda Reforma de Salud: Aseguramiento y compra-venta de servicios. Salud Colectiva [Internet]. 2010 [revisión 2019 oct. 17];6( 2 ):137-148. Disponible en: http://www.s cielo.org.ar/scielo.php?script=sci_arttext\&pid=S1851-82652010000200002\&lng=es.

8. Uribe M, Abrantes R. Las reformas a la protección social en salud en México: ¿rupturas o continuidades? [Internet] Perf. Latinoam. 2013;21(42):135-162 Disponible en: http://www.scielo.org.mx/scielo.php ?script $=$ sci_arttext\&pid $=$ S0188-76532013000200006

9. Salud Pública de México. Entrevista a la Dra. Asa Cristina Laurell, Secretaria de Salud del Distrito Federal. Salud Pública Méx [Internet]. 2005 feb. [revisión 2019 oct. 17]; 47(1):82-86. Disponible en: http://www.scielo.org.mx/scielo.php?script=sci_arttext\&pid=S0036-36342005000100013\&lng=es

10. Ocampo-Rodríguez MV, Betancourt-Urrutia VF, Montoya-Rojas JP, Bautista-Botton DC. Sistemas y modelos de salud, su incidencia en las redes integradas de servicios de salud. Rev. Gerenc. Polit. Salud. 2013;12(24):114-129

11. Freitas R, Gomes A, Ferreira M, Chagas I. Percepção dos profissionais sobre a coordenação entre níveis de atenção à saúde em dois municípios pernambucanos de grande porte. Interface (Botucatu). 2018;22(65):423-434. 
12. Pan American Health Organization. Renewing primary health care in the Americas. Concepts, policy options and a road map for implementation in the Americas. Washington: OPS; 2010.

13. Reid R, Haggerty J, McKendry R. Defusing the confusion: concepts and measures of continuity of healthcare. Ottawa: Canadian Health Services Research Foundation; 2002.

14. Vázquez M, Vargas I, Unger J, Mogollón A, Silva M, Paepe P. Integrated health care networks in Latin America: toward a conceptual framework for analysis. Rev. Panam. Salud Públ [Internet]. 2009;26(4). Disponible en: https://scielosp.org/article/rpsp/2009.v26n4/360-367/

15. Vázquez ML, Vargas I, Mogollón-Pérez A, Ferreira da Silva M, Unger JP, De Paepe P, editores. Redes Integradas de Servicios de Salud en Colombia y Brasil: Estudio de casos [Internet]. Bogotá: Editorial Universidad del Rosario; 2017. http://www.doi.org/10.12804/tm9789587388121

16. México. Dirección General de Información en Salud. Proyecciones de la Población por condición de derechohabiencia, 2010-2018. Ciudad de México: Secretaría de Salud; 2010.

17. Dirección General de Información en Salud. Catálogo de Clave Única de Establecimientos de Salud (Clues) [Internet]. Ciudad de México: Dirección General de Información en Salud; 2020 [revisión 2020 feb. 10]. Disponible en: http://www.dgis.salud.gob.mx/contenidos/intercambio/clues_gobmx. html

18. Vargas I, Mogollón-Pérez A, De Paepe P, Ferreira M, Unger JP, Vázquez ML. Barriers to healthcare coordination in marketbased and decentralized public health systems: a qualitative study in healthcare networks of Colombia and Brazil. Health Pol Planning. 2016;31:736-748.

19. Vargas I, Garcia-Subirats I, Mogollón-Pérez A, Ferreira M, Eguiguren P, Cisneros A, et al. Understanding communication breakdown in the outpatient referral process in Latin America: a crosssectional study on the use of clinical correspondence in public healthcare networks of six countries. Health Pol Planning. 2018;33:494-504. https://www.doi.org/10.1093/heapol/czy016

20. Miranda-Mendizábal A, Vargas I, Mogollón-Pérez AS, Eguiguren P, De Medeiros Mendes MF, López J, Navarrete M. Conocimiento y uso de mecanismos de coordinación clínica de servicios de salud de Latinoamérica. Gaceta Sanitaria [Internet]. 2018. Disponible en: https://www.sciencedirect.com/sci ence/article/pii/S0213911118302462

21. Ferreira C, Chagas I, Ferreira M, Vargas I, Vázquez ML. Conocimiento y uso de mecanismos para la coordinación clínica entre niveles de asistencia en dos redes de atención de salud en Pernambuco, Brasil. Cad. Saúde Pública. 2019;35(4):e00119318

22. Ferrada O, Méndez C. Implementación de las redes asistenciales de salud en Chile: percepciones de los profesionales de la salud. Rev. Gerenc. Polit. Salud. 2013;12(24):100-113.

23. Vázquez M. Vargas I, Terraza R, Pizarro V. Marco conceptual para el estudio de las organizaciones sanitarias integradas. Organizaciones sanitarias integradas. Un estudio de casos. Barcelona: Consorci Hospitalari de Catalunya; 2009.

24. Vargas I, Vázquez M. Análisis comparativo. Organizaciones sanitarias integradas. Un estudio de casos. Barcelona: Consorci Hospitalari de Catalunya; 2009.

25. Terraza R, Vargas I, Vázquez ML. La coordinación entre niveles asistenciales: una sistematización de sus instrumentos y medidas. Gac Sanit. 2016; 20(6):485-495. 
Angélica Ivonne Cisneros Luján / Dulce María Cinta Loaiza /

María Alejandra Sánchez Bandala / Vianey González Rojas

26. Puccini PT, Keddy-Cornetta V, Zogbi-Sahyom T, Pagliarini-Fuentes IC, Gimenez-Botta LM, FioriniPuccini R. Concepção de profissionais de saúde sobre o papel das unidades básicas nas redes de atenção do SUS/Brasil. Ciência Saúd Colet. 2012;17(11):2941-2952.

27. Henao D, Vázquez ML, Vargas I. Factores que influyen en la coordinación entre niveles asistenciales según la opinión de directivos y profesionales sanitarios. Gac Sanit. 2009;23(4): 280-286.

28. Ávila AY, Lima GV. Retinopatía que amenaza la visión en pacientes diabéticos no referidos al oftalmólogo. Gac Méd Méx. 2013;149:624-629.

29. Menéndez EL. Modelo médico hegemónico, crisis socioeconómica y estrategias de acción del sector salud. Cuad Méd Soc. 1985;33:33-34.

30. Rodríguez BRA, Reynales SLM, Jiménez RJA, Juárez MSA, Hernández AM. Costos directos de atención médica en pacientes con diabetes mellitus tipo 2 en México: análisis de microcosteo. Rev Panam Salud Publica. 2010;28(6):412-420.

31. Martinussen P. Referral quality and the cooperation between hospital physicians and general practice: The role of physician and primary care factors. Scand. J. Public Health. 2009;41:874-882.

32. Vargas I, Mogollón PAS, De Paepe P, da Silva MR, Unger JP, Vázquez ML. Do existing mechanisms contribute to improvements in care coordination across levels of care in health services networks? Opinions of the health personnel in Colombia and Brazil. BMC Health Serv Res [Internet]. 2015;15:213. Disponible en: https://bmchealthservres.biomedcentral.com/articles/10.1186/s12913-0 $15-0882-4$

33. Sansó FSJ. La referencia y la contrarreferencia como expresión de la interrelación policlínico-hospital. Rev Cub Med Gen Integr [Internet]. 2002;18(3). Disponible en: http://scielo.sld.cu/scielo.php?scrip $\mathrm{t}=\mathrm{sci}$ _arttext\&pid=S0864-21252002000300015

34. Aller MB, Vargas I, Sánchez I, Henao D, Coderch de Lassaletta J, Llopart JR, et al. Continuity of Care between Levels Perceived by Users of the Health System in Catalonia, Spain. Rev. Esp. Salud Pública. 2010;84(4):371-387.

35. Rubio AV, Rodríguez IML, Sampedro ME, Victores BC, Alechiguerra A, Barrio GJL. Evaluación de la calidad de comunicación entre niveles asistenciales mediante el documento interconsulta. Atención Primaria. 2000;26(10): 681-684.

36. Von Bültzingslöwen I, Eliasson G, Sarvimaki A, Mattsson B, Hjortdahl P. Patients' views on interpersonal continuity in primary care: a sense of security based on four core foundations. Fam Pract. 2006;23:210-219.

37. Waibel S, Henao D, Aller MB, Vargas I, Vazquez ML. What do we know about patients' perceptions of continuity of care? A meta-synthesis of qualitative studies. Int J Qual Health Care. 2012;24:39-48.

38. Llanusa SB, Rojo NC, Hernández M, Capote MR, Pérez J. Las tecnologías de información y comunicación y la gestión del conocimiento en el sector salud. Rev Cub Salud Pública [Internet]. 2005;31(3). Disponible en: http://scielo.sld.cu/scielo.php?script=sci_arttext\&pid=S0864-346620050 00300008 
39. Díaz NJ, Guinart ZN. Las urgencias en la atención primaria. Utilización del servicio. Rev Cubana Med Gen Integr [Internet]. 2007;23(4). Disponible en: http://scielo.sld.cu/scielo.php?script=sci_arttext\& pid $=$ S0864-21252007000400002

40. Bermejo JC. Atención centrada en la persona. Rev. Chil. Endocrinol. Diabetes. 2015;8(3):121-122.

41. Walsh J, Harrison J, Young J, Butow P, Solomon M, Masya L. What are the current barriers to effective cancer care coordination? A qualitative study. BMC Health Serv. Res. 2010;10:132.

42. Báez MJM, Sánchez OA, Garcés RG, González CR, Santos BL, López CR. Motivos y condicionantes de la interconsulta entre atención primaria y especializada. Semergen. Med Famil. 2012;39(2):89-94.

43. Moreno ME. ¿Y si adaptáramos los servicios hospitalarios de urgencias a la demanda social y no a las necesidades de salud? Emergencias. 2008;20(4):276-284.

44. Hernández HIL, Mercado MFJ. Estudio cualitativo sobre la atención médica a los enfermos crónicos en el Seguro Popular. Salud Pública Méx [Internet]. 2013 abr. [revisión 2017 mar. 31];55(2):179-184. Disponible en: http://www.scielo.org.mx/scielo.php?script=sci_arttext\&pid=S0036-3634201300020 0009\&lng=es

45. Fernández MA, García GJL, Palmero PC, García VMB, Páez PJM, Álvarez AR, et al. Continuidad asistencial. Evaluación de un programa de colaboración entre Atención Hospitalaria y Atención Primaria. Revista Clín Esp. 2007;207(10):510-520.

46. Corrales ND, Alonso BA, Rodríguez LMA. Continuidad de cuidados, innovación y redefinición de papeles profesionales en la atención a pacientes crónicos y terminales. Informe SESPAS 2012. Gac Sanit. 2012;26(1):63-68.

47. Tabarquino R, Pulgarin D, Giraldo A. Dinámica de una red integral de prestadores de servicios de salud (RIPSS). Rev. Gerenc. Polit. Salud. 2018;17(34):1657-7027.

Notas

* $\quad$ Artículo de investigación 